\title{
OPEN
}

\section{4 \\ States of Rest: Interdisciplinary Experiments}

\begin{abstract}
This chapter represents a hinge in the book, moving from the more pragmatic material in its first half to the more conceptual work that we take up in the second half. The chapter is centred around the interdisciplinary project from where we both write - Hubbub, a Wellcome Trust funded project that opens up 'rest' as a simultaneously biological, psychological, social, historical, and cultural object. The chapter offers two accounts of how Hubbub came about, focusing first on individuals, and their shared work, and, second, on the institutions and funders that have intersected with that work. The chapter closes by making clear some of the intellectual tensions that nonetheless run through Hubbub, as it works through different registers of intellectual practice.
\end{abstract}

Keywords: collaboration; Hubbub; interdisciplinarity; neuroscience; rest; social science

Callard, Felicity and Des Fitzgerald. Rethinking Interdisciplinarity across the Social Sciences and Neurosciences. Basingstoke: Palgrave Macmillan, 2015. DOI: 10.1057/9781137407962.0007. 


\section{Introduction}

We have been asked, many times, by researchers of all stripes, how we have put our 'interdisciplinary projects' together. In this chapter, we focus neither on particular elements or episodes of interdisciplinary working (see Chapters 1-2), nor on the broader problematics that are reshaping the landscape of interdisciplinary research that addresses the 'bio' and the 'social' (see Chapter 3), but instead give some sustained attention to how one large interdisciplinary project, Hubbub, directed by FC (and in which DF is one of many collaborators) came into existence. In what follows, we describe the transformation of a number of nascent, fragmentary germs of ideas into a large, unruly collaborative and ongoing - project, which is investigating rest and its opposites in neuroscience, mental health, the arts and the everyday, and which is based in The Hub at Wellcome Collection from October 2014 until July 2016. (The project was the outcome of a specific, innovative grant call from The Wellcome Trust for interdisciplinary research projects addressing health and wellbeing to apply to inhabit the specially designed space, 'The Hub', for 22-month residencies. Hubbub was selected through a competitive application process as the first residency. For additional contextual details, see Callard, Fitzgerald, and Woods 2015.)

Hubbub offers a crucible in which we might transform our observations and prescriptions about interdisciplinarity across the social sciences and the neurosciences, which we set out in this volume, into a live, interdisciplinary project - as well as in which we might observe the differences and continuities between our plans and prescriptions, and their implementation through material spaces and topologies, particular people, and a given time period. The account that follows is one that has not been shaved clean of texture, abrasions, frayings, and fractures. (We stress it is very much an account diffracted through the interests and memories of the two of us; the co-investigators of the project, as well as the many other collaborators, would undoubtedly provide very different accounts.) Our intent is to thread some of the preoccupations of this book - vis-à-vis spaces and geographies, power, affect, and heterogeneous modes of intervention - through our narrative so that you might adjudicate what purchase these thematics might have when translated into an experimental entanglement over which one of us, at least, as Principal Investigator, has significant control. 


\section{Rest}

This volume has been assembled in the open-plan Hub at Wellcome Collection - which provides time and space to consider how Hubbub (as a project with its own organizational logics), is curating, limning, and analysing the various matrices and topologies (see Chapter 5) that characterize the networks of human and non-human agents that comprise it. The award of the first residency of The Hub (to Felicity as Principal Investigator and the four co-investigators [Charles Fernyhough, Claudia Hammond, Daniel Margulies, and James Wilkes]) has brought with it the opportunity to implement a different model of interdisciplinary neuro-social science from many we discuss in the course of this volume. Before we narrate the coming together of Hubbub, we want briefly to outline some of the research trajectories that Hubbub is taking, and the experimental entanglements it is in the process of bringing into being.

As a project tracking 'rest' through a range of simultaneously biological, literary, psychological, sociological, clinical, historical, and cultural iterations, Hubbub comprises a thick assemblage of interdisciplinary projects and questions. These include, to take a quick sample: (1) Focusing on the ways in which daydreaming - as phenomenon, construct, and trope - has meandered across a number of disciplines within its own history. Hubbub researchers are pursuing both sociological accounts of, as well as psychological and neuroscientific investigations into, mind-wandering and daydreaming, to focus, in particular, on the states to which daydreaming has been (or might be) opposed or related. Central, here, are attempts to investigate not only how varied forms of experimental practice (across different disciplines) elicit, construe, and represent daydreaming, but how such practices might cross-fertilize methods used in other domains. (2) Thinking, working with, practising, and experimenting with exhaustion - and, in particular, cleaving exhaustion from its usual intimacy with the subjective phenomenon of 'tiredness', and with the overworked figure of a tired individual's body and mind. Archival work, poetry, critical-creative writing, and cultural-philosophical analyses are central to our investigations into exhaustion - not least vis-à-vis how they might complicate psychiatric and other clinical accounts of this bodily state. (3) Thinking, beyond commensensical assumptions, about the ways in which people experience, conjure, and imagine rest, as an abstract phenomenon, 
but also as an index of the quality of their own daily lives. Large-scale surveying as well as public engagement are central to Hubbub's work in this domain. (4) Focusing on cartographies of rest, to ask both how particular spaces get imagined as noisily restless, and what kinds of technologies we can bring to bear on how those spaces are actually experienced. Researchers are using sociological and anthropological methods to investigate these questions, as well as experimenting with self-tracking devices that will draw together physiological, phenomenological, and geographical data.

One of the foremost intellectual preoccupations of Hubbub is an interdisciplinary attention to the brain and mind at rest. This attention has roots in the previous collaborative work of Daniel Margulies, Jonathan Smallwood, and FC (Callard et al. 2013; Callard and Margulies 2011, 2014; Callard, Smallwood, and Margulies 2012), which has spiralled in and out of cognitive neuroscience, cognitive psychology, and the history of science - and which is being extended, empirically and conceptually, in Hubbub. Meanwhile, preoccupations and priorities from other disciplines and disciplinary debates, as well as from other modes of inquiry, have gradually come into view. This latter category, for instance, includes a collaboration involving Charles Fernyhough (CF), Simone Kühn (SK), Russ Hurlburt and others, which we discussed in Chapter 2 (in the section on 'Co-organizing'). This research, which brings together phenomenologically rich data from Descriptive Experience Sampling, and cognitive neuroscientific data on research participants' brain states, so as to elicit, capture, and visualize 'inner experience' (Kühn et al. 2014), has brought to visibility compelling insights and empirical findings that offer new ways of understanding the brain and mind 'at rest'. Among these insights is an investigation of the limitations of common ways of investigating 'spontaneous thought' and 'inner experience' (e.g. those that rely on respondent questionnaires), as well as the need to attend more carefully to phenomenological experiences of mental and bodily 'rest' (or lack of rest) while in the scanner (Kühn et al. 2014). CF, meanwhile, directs another large interdisciplinary project (Hearing the Voice). There are not only substantive intersections between the two networks of collaborators, but Hubbub, as it took form on paper as a grant proposal, was - happily - able to draw on some of the interdisciplinary methods and structures that were already being tried out within Hearing the Voice. 
New collaborations within Hubbub intersect with these long-developing groups and methods. One collaboration with co-investigator James Wilkes - a poet and writer with long-standing interests in the intersections of poetry and cognitive science (Wilkes and Scott forthcoming) was started because of the lure of The Hub award, and was facilitated by interests that both CF and James Wilkes have long had in the voice. Wilkes (together with several of his collaborators from the arts [who include writer and performer Holly Pester, composer Antonia Barnett McIntosh and poet Steven Fowler]) has been opening up attention to the mind and body at rest, via considering the roles of pause, silence, noise - not least in the ways in which they loop between voice, body, and mind (Wilkes 2015) - in critical-creative literary production, as well as in performance and composition. Co-investigator Claudia Hammond, with her long-standing career as a broadcaster and writer, has been interested in exploring how the experiments on rest taking place within The Hub might draw in the perspectives and reactions of various publics beyond it. Hilary Powell, a medieval historian, is interested in exploring the structural similarities and dissimilarities between monks' attempts to keep themselves from being distracted during the labours of devotion and current psychological models of mind-wandering and inner speech. Josh Berson, an anthropologist of human-material interfaces, is working to chart the technosomatics of restful space (Berson 2015). DF, meanwhile, is working to bring his interests in the long histories of biosocial accounts of cities, mental health, and stress (see Chapter 3, 'The urban brain') into contact with other collaborators' interests in restful and restless cities (Fitzgerald, Rose, and Singh forthcoming); Lynne Friedli has been tracing the experiences of psychological coercion amongst those who are unemployed, whereby what might previously have been characterized and experienced as periods of 'idle rest', are now stuffed full with corrosive demands for self-actualization if one is not to lose one's social security benefits (Friedli and Stearn 2015). Ayesha Nathoo, a cultural historian, is analysing various twentieth-century practices of relaxation. (There are multiple, additional collaborators whose research we have not picked out here.) As the Hubbub collaborative network has expanded (see Hubbub 2015), this much larger group has poured intense energy into thinking carefully about the various ways in which rest, relaxation, bodily posture, and mental quietude have been - and continue to be construed, represented, spatialized, and studied in medical, scientific, social, and cultural domains. 


\section{The origin story: version 1}

We want to draw particular attention, in this chapter, not to what this project is doing, but how it came about. Here is one origin story. Not unsurprisingly, it construes one of the authors of this book as one of the central protagonists, and centres on a tight-knit group of surrounding characters. In 2008, FC was immersed in one of the heartlands of psychiatric, psychological. and neuroscientific research. She was also experiencing a certain amount of epistemological and professional disquietude. First, there was the unease she felt at how her role as a social scientist - and specifically as a person whose interests were those of the psychiatric 'service user' - was being imagined within a large programme of research addressing translational mental health. In this role, FC was someone who, she felt, was often positioned as simply assisting clinical and other academic mental health researchers with smoothing the 'translational' pipeline, or with 'understanding the patient [or family] perspective' (Callard, Rose, and Wykes 2012). She was considered rather less frequently, she believes, as a researcher whose expertise in social theory and the history of psychiatry might shift some of the logics at work in the translational endeavour ( $\mathrm{Mol}$ 2002). Second, she was curious about the complexity of the genetic and neurobiological accounts that colleagues advanced in seminars and in the collaborations that she was being drawn into (see e.g. Greenwood et al. 2011) - accounts that challenged her existing, rather static assumptions about the 'biomedical'. In the midst of this intellectual quandary, she received an email inviting applications for the first European Neuroscience and Society Network interdisciplinary 'Neuroschool', a five-year programme 'involving leading neuroscientists and social scientists from eleven European countries in collaborative research and debate. The Neuroschool was intended to 'foster learning in an interdisciplinary symmetrical environment', taking behavioural genetics as its entry point. Current methodologies of experimentation (as well as the implications thereof within society), the 'latest scientific evidence in the field', and 'the history and sociology of psychotropic drugs' were all scheduled for discussion and debate (European Neuroscience and Society Network, cited in Costandi 2008; see also Frazzetto 2011 for a comprehensive account of the pedagogies involved within the Neuroschool).

On attending the Neuroschool, FC came to know a cognitive neuroscientist through jointly engaging in experimental practices that are used 
in behavioural genetics and in which neither was an expert. In FC's mind, there was - as we noted in Chapter 2 ('Co-authoring') - no particular emergence of a topic or problem that could form the basis of an interdisciplinary collaboration' between the two of them. Over time, however, they pursued a number of investigations of the history and the present of the field of research in which the neuroscientist was embedded, namely that of resting state fMRI (rsfMRI) research. (While the origins of any field are always contested, resting state fMRI is commonly understood to have emerged out of work done in the mid- to late-199os by Bharat Biswal and colleagues on functional connectivity (Biswal et al. 1995), and Marcus Raichle, Debra Gusnard and colleagues on the default mode of the brain (Gusnard, Raichle, and Raichle 2001; Raichle et al. 2001)). FC, with long-standing interests in the potency of mind-wandering and fantasy, and the varied socio-spatial settings in which they might take place (Callard 2013), was unsure how 'rest' and 'resting' were being conceptualized and modelled within resting state fMRI. Both she and her collaborator became intrigued, indeed, by how the growth in resting state fMRI had been accompanied by a rise in neuroscientific interest in phenomena such as mind wandering and daydreaming that had previously been consigned to the margins of cognitive psychology.

This collaborative enterprise was provided with greater consistency by their committing to co-author an article in a special issue of a social theory journal. The paper, FC recalls, passed through thirty or so drafts, mostly sent to and fro in the early hours of the morning - the hours that comprised a window of time before one of them went to bed and after which the other had risen. Both of them, FC surmises, were able to carve out pockets of time to pursue their collaborative endeavour, outside of any formal support or financial structures, in part because they were at a relatively early stage of their careers. They also found a creative way to work with the exigencies and idiosyncrasies of geographical distance and their different quotidian patterns. Indeed, FC believes that their diverse phenomenological and material experiences of 'rest' (and lack of rest) ended up inflecting - obliquely - the kinds of scientific and normative questions they were interested in pursuing within their collaboration. (Such as: Are there better or worse ways 'to rest'? How do bodily and mental states of exhaustion intertwine with one another? Has the scientific literature embedded normative assumptions into models of 'the resting state' and of mind-wandering?) Dynamic material and affective contours - those that enjoin mind and body, and those that allowed 
precarious intersections between two researchers writing, together, across significant geographical distances - were (again, this is only FC's account) at the heart of, rather than epiphenomenal to, their interdisciplinary collaboration.

If FC and her collaborator's first published co-authored paper could be said to constitute the formal imprimatur for their collaboration, it should not be overemphasized as the thing that consolidated an interdisciplinary problematic that could function as a goad, at least for FC, for further collaborative work. (Here, we note in passing the mess of what goes on behind the scenes of a collaboration. What should count as an 'event' or 'output' to be remembered, and noted, and given weight when tracing twists or turning points in the development of an interdisciplinary collaboration?) FC and her collaborator's shared interest in psychoanalysis (a profoundly marginalized field within the cognitive neurosciences) led them to a short opinion piece by cognitive neuroscientist and psychoanalyst Peter Freed in which Freed attempted to carve a place for psychoanalysis within the still young (interdisciplinary) field of resting state fMRI by tying the 'mind at rest' to the psychoanalytic problematic of 'free association'. 'Functional neuroimaging's interest in free association', Freed argued, 'seems to be happening in a historical vacuum, at least for the majority of researchers'. In particular:

Freud and the couch are nowhere mentioned, though the similarities to psychoanalytic practice are rather striking: the subject enters the room, lies down, and has his or her head examined by a largely silent clinician using an opaque technology as he or she thinks about 'whatever comes to mind'.... But there is a sharp difference between the two fields. In psychoanalysis, exquisite attention is paid to each twist and turn of thought.... This and any other content-laden chain of thinking is entirely hidden in resting-state studies, where the moment-to-moment cognitions and emotions are invisible, unrecorded; all that is known is that the subject is 'at rest' or 'in default mode.'... Without a task to model in the data analysis, all that can be said of the period of free association is that the subject was 'ruminating.' Do we learn anything from this? (Freed 2008, 102)

A moment of conceptual clarity emerged for FC: this was the point - a moment of responding in her head to a short piece of writing that would likely be read by few and likely cited by even fewer - that she remembers the stakes of her own interdisciplinary desires coming into focus. Freed's intervention, on behalf of psychoanalysis, made it clear that it was, at that historical moment, by no means wholly settled how the 
relationship between brain dynamics (the province largely of cognitive neuroscientists) and psychological processes (the province largely of those trained in the 'psy disciplines' (Rose 1996)) would be understood and consolidated in coming years. FC - through engaging extensively with her collaborator to understand more about how, at the heart of the resting state field lay the problem of relating brain dynamics to processes of thinking per se - realized, then, that there was a great deal at stake in terms of which psychological models, and which means of eliciting 'inner experience' would end up becoming embedded in an expanding field. It was not preordained, she realized, that neuroanatomical investigations of the brain's connectivity should be sutured together with the working objects and current experimental paradigms of cognitive psychology.

Here, then, was an opening for the interpretive social sciences, humanities, and the arts practically to intervene in - i.e. to contribute to making - a sub-field, rather than remain an external adjudicator of its progress and insufficiencies. FC returned to late nineteenth- and twentieth-century philosophy, psychoanalysis, and psychology, so as to think how it might be possible to open up other paradigms through which to investigate mind-wandering and similar phenomena experimentally. A fortuitous move to the city near where her collaborator was based provided favourable institutional conditions, as well as intimacy with researchers from a range of disciplinary backgrounds, to pursue those lines of investigation. Simultaneously, a new collaborator appeared on the scene, a psychologist ready to reach out as a scientific collaborator to FC's neuroscientific interlocutor. In time, all three of them started experimenting jointly, both on conceptual interventions within the resting state field, and on scientometric investigations of the emergence and organization of the various sub-fields exploring resting state research. Such a process involved FC's collaborators generously introducing her to new methods and new ways of thinking, which significantly shifted her existing understandings of the history of daydreaming, mind-wandering and related phenomena. Through such joint experimentation, the familiar landscape - one in which the social scientist or humanities scholar simply depicts and analyses the experimental work of her scientific colleagues - was decisively tilted, and a new, uneven topology of collaboration and of epistemological exchange opened up. FC's capture by, and fascination with, new modes of working and thinking together across the disciplines were, in turn, fundamental to how she approached the development of the Hubbub project. 


\section{The origin story: version 2}

Version 1 centred largely on persons and their interests, but was, in general, uninterested in material or institutional questions. The focus was on ideas, on conundrums, and on people figuring out new ways of working together to address those conundrums. (We nodded briefly towards the importance of temporal congruence for the writing of collaborative papers, and the serendipity of changes in geographical locations.) In placing centre stage a search for intellectual quarry, whose hue became clear only in the chasing, we have perhaps repeated a well-worn story about interdisciplinarity in which nothing much appears outside of the disciplinary and interdisciplinary passage of ideas. Let us try again, then, in thinking about what matters, by providing a more topologically sensitive account of some of the researchers, technologies, organizations, funders, and media that comprise the scene of Hubbub.

Many of the researchers who appear in this book (either explicitly, as characters, or unnamed, as delegates at the various workshops we have mentioned) were, at least at the start of the interdisciplinary projects described, precariously employed on fixed-term contracts, who were willing (or desperate) enough to move between cities and countries in Europe and North America. Such moves were not, we conjecture, simply to find the next intriguing disciplinary location (though we are aware of several disciplinary 'transfers' amongst our broad network of collaborators, which include: from cognitive neuroscience to anthropology; from geography to the history of science; and from Continental philosophy to cognitive neuroscience). Of greater salience, we think, was their need to offer themselves up to a wide geography in which their labour power might be bought and sold. If interdisciplinarity demands the co-location of researchers with different sets of disciplinary expertise, then the stressful nature of the academic labour market - characterized by frequent mobility and sequential fixed-term contracts - is no minor protagonist. Crossings - as the literatures on mobility and migration have for a long time made clear - involve the complex and ambivalent transfer of cultures, methods, techniques, fantasies, and habits. Affects are twisted and crushed - enfolding gratitude, ambivalence, and resentment within and between bodies. Indeed, a number of our papers that we have cited in this book were written in and through - at least for the two of us varying states of distress: interdisciplinary co-authorship can function as an inadequate salve to various kinds of wounds, as well as a way of 
extending and sustaining sociality. Many of our collaborations, too, have been facilitated by collaborators' material and intellectual generosity in hosting one or both of us, or of enabling those geographical moves to happen in the first place. These larger stories of material and cultural inequalities, and of complex networks of hospitality, are perhaps far more significant, in anatomizing interdisciplinary collaborations, than the epistemological and disciplinary inequalities that we will discuss in Chapter 6. It is important to emphasize that neither material inequalities, nor host-guest relationships, line up neatly on disciplinary lines.

What of institutions and funders? The decision by the National Institute for Health Research (NIHR), which was created in the United Kingdom in 2006, to fund multi-disciplinary biomedical research centres for translational research (Snape, Trembath, and Lord 2008) is important, here. One of us (FC) was able to move from being largely external to the psychiatric and psychological enterprise (in an earlier research career as a historical geographer of psychiatry) to sitting at the heart of it, via the imperative not only to bring sociological perspectives to neuroscientific and genetic/genomic research, but to ensure that 'patient/service user' perspectives were heard. We also emphasize the emergence of research centres challenging the decades-long standoff between large parts of the sociological and the biological. The BIOS centre at the London School of Economics (established and directed by Nikolas Rose, which has now become the Department of Social Science, Health \& Medicine at King's College London), and the Interacting Minds Centre at Aarhus University (directed by Andreas Roepstorff) have been foundational to the work that we and many of our collaborators have carried out.

A crowded and jostling international university environment, in which there are increasingly intense demands to acquire external grant funding, has also helped foment interest in establishing cross- and interdisciplinary projects that sit outside of particular departments and disciplines. This demand has dovetailed with the decision by major funders (e.g. The Wellcome Trust and the Volkswagen Foundation) to develop funding streams that would support research programmes bringing humanities scholars and interpretive social scientists into much closer proximity with life scientists. We also need to mention, at least for our own particular trajectories, the invigoration of the field and purpose of medical humanities within the United Kingdom, which is also, in part, a story about the reconfiguration of the favoured place of medical history 
and of medical sociology in relation to biomedicine. For the emergence of Hubbub, the Centre for Medical Humanities at Durham University where FC took up a permanent position in $\mathbf{2 0 1 2}$ - is important as a node in an increasingly international network of interdisciplinary centres advancing collaborative work across the life sciences, health sciences, humanities, and social sciences (e.g. Atkinson et al. 2015; Viney, Callard, and Woods 2015).

Finally, it is no coincidence that the accounts of emerging interdisciplinarity that we have given in this chapter, and throughout this volume, move in lockstep with a rapidly changing and increasingly dense technological ecology for collaborative writing and thinking. Such an ecology is characterized by divergent temporalities and purposes to which each part of it is put to use. The changing shape of this ecology has been central to helping open out possibilities for collaboration - across geographical and disciplinary terrains - whose trajectories we have described in this chapter. FC remembers the first time she completed a co-authored article with a scientific collaborator, in which Dropbox (a file hosting service, introduced to her by her collaborator, that provides cloud storage and file synchronization), to her, seemed almost like a third author; for her collaborator, on the other hand, she inferred that this was a technology that was already so assimilated that it was on the point of being old-fashioned. While scholars such as Ben Kafka have analysed the historical role and nature of paper as central to the operations of bureaucracy (Kafka 2012), it is not clear whether there is, yet, any adequate account of how intimately current practices of writing, talking, storing, tracing, joking, and fighting through and across platforms such as Google Hangouts and Google Docs, Skype, Slack, Dropbox, Twitter, Zotero, and so on are bound up with the changing shapes of collaboration, non-collaboration and interdisciplinarity. It has been striking, in the trajectories that built towards Hubbub (as well as those ongoing dynamics within it), to note how there is no straightforward way in which one can map ease and interest in the use of particular media on to particular disciplinary allegiances. Here, then, unexpected media topologies - in which collaborators from very different disciplines might come together around common commitments to particular ways of sharing, communicating, or keeping to oneself - are produced that run counter to usual stories of epistemological differences that require a 'reaching across' disciplinary boundaries. How both we and our collaborators have agreed (keenly or reluctantly), or not, to communicate via particular kinds of 
media has told us a great deal, not only about the heterogeneity of intraas well as interdisciplinary commitments and backgrounds, but about different visions of what collaboration, loss of control, exchange, shared labour, incorporation of ideas, and writing, itself, might mean.

\section{'We've now split ...'}

In presenting, here, particular narratives of how things emerge, we might have given the (false) impression that the hard work of establishing, and persisting with, interdisciplinary collaborations was largely in the (pre-Hubbub) past. Let us emphasize, then, in closing, the ongoing challenges that characterize any space in which collaborators with very different sets of expertise, as well as different understandings and practices of interdisciplinarity, work together. There is, we both believe, no singular direction of travel within Hubbub. Our collaborators do not have a shared vision of what the purpose or telos of interdisciplinarity might be, nor what the appropriate working methods are for engaging in such interdisciplinary collaboration. They - and we - are undoubtedly working with a variety of needs, logics, ontologies, and practices (Barry, Born, and Weszkalnys 2008). Consider this short note, taken by one of the present authors (DF) during an early project meeting (all errors of typography and syntax are from the original):

- Kind of feels like the psychologists are having a conversation among themselves.... - what happened to the larger questions we started with? This is how it tend to go; we start off with something high-level, then the psychologists start talking through what they imagine to be the pragmatics... and suddenly we're having a conversation about the parameters of having a psychological study.

- The issue $i$ have is that in rooms that are dominated by psychology/ psychologists ( $>50 \%$ in this room) $i$ always feel obloged to represnet and then get fiured as representing - all the things that are not psychology. And that's an impossible position, so you aways end up syang very broad \& silly things.

- We've now split [...] [Person X] has said that what holds [out half of the split] together is the willingness to defer ontological commitments, which $i$ thnk he's basically right about - but this seems to have caused a little bit of confusion controversy. 
[...]

- afte lunch we;ve split - the psychologsts have all [gone] to work [on their own] - te rest of us have have stayed in the room to [...] The thing is totally split at this point. [Person Y] asks (rightly) what does it mean for the project that we've all split off like this??

- [Later, Person Z] interestingly insisting that if we force a top-dwon interdisicplinarity, it'll be a total balls (and why we've been having this endless discussion [...] going around in circles); the best thing is to let people just do what they're good at, and then (a) trust ID [interdisciplinary] stuff to bubble up from the talented poeple we have;

(b) have some curation at the top.

What to make of this? For a start, let's notice how epistemological and ontological difference plays out spatially (some collaborators physically leave the room), affectively (note the documenter's own irritation, as well as the implication that those moments of controversy have become fraught across the whole group), and through an unequal dynamics of epistemological power (i.e. psychology's perceived chauvinism, which, on the documenter's account, allows a rapid pull away from the 'highlevel' and a turn to the 'parameters of [... ] a psychological study'). What rings out from the note is the author's jaundiced account of what is at stake for psychologists - as well as his juvenile sense of frustration at being part of a conversation that felt as though it were dominated by the normative commitments of that discipline. But if the details are fuzzy (in fact, not all scientists left the room), the feelings expressed here are real enough. The point is not about 'psychology' or 'psychologists' (a category, in any event, poorly described in this note) but about some in-the-moment sensations through which logics of collaboration, even in as tight an assemblage as this one, are both produced and experienced. Needless to say, the collaborators positioned as 'psychologists' (they were not even all psychologists) have their own accounts of this episode. As one put it to us, on reading the above extract:

It was a very productive and interesting discussion, although I remember being powerfully reminded of some of the disciplinary differences. I like to think that we psychologists are aware of the endless possibilities for defining and critiquing terms, but it's also true that our instinct is to get on and do stuff, even if the conceptual framework is not perfect. I remember thinking, My God, how does anything ever get done, if we're going to argue at every step about whether everything we want to say is equally applicable to all strata of society? How do these (lovely, smart) people ever finish anything?! 
Another psychologist who was present on the day remembers it like this:

My recollection of the day is that after hours and hours of nothing getting decided, and this being our only chance all together to get the planning done that the whole group decided the only way to do it was to split into two groups, but that's just my memory of it ... Of course I do see that the problem for interdisciplinarity here was that all the psychologists chose the practical planning group and everyone else chose the discussion group. So we all conformed to the various stereotypes. I do have to wonder whether the [project in question] would be at the advanced stage that it's at today, if the group had stayed as one?

Here then, even as the three accounts diverge markedly in what happened, there is, at least to our eyes, a way in which the discipline of psychology, here, is positioned - or imagines itself to be - as more pragmatic than the other disciplines. We are reminded that, even in Hubbub, which is an endeavour in many ways defined by its successes, disagreement abounds. Feelings can run high. Very different ideas of the stakes of the project, as well as the modes of investigation appropriate to it, run headlong into one another. If the split in the above meeting was temporary, and indeed productive, we believe, for the research of Hubbub as a whole, we remind ourselves of the thin sutures that continue to hold interdisciplinary collaborative projects together. We remain deeply alive to our own capacity, at any moment, to come undone.

\section{Notes and Queries: 4}

Q: What is the most important element of an interdisciplinary project? The idea? The people? The funder? The level of institutional support? What should I be really looking for - an excellent proposal or a brilliant collaborator?

This is an area where our experience tends to run against the usual advice. Typically what people will say is that the problem should come first. And so, good interdisciplinarity happens when, for example, a philosopher has a conception of free will that can be empirically tested, and he (in analytic philosophy, it almost certainly is a he) runs into a neuroscientist who has methods that can perform that empirical test - and who, in turn, has some ideas about free will that could do with some conceptual refinement. This 
is fine, of course - but like so much else in the interdisciplinary field, it is predicated on a potentially unrealistic idea of how intellectual work actually happens. FC and her neuroscientific collaborator started working on rest, for example, because they liked one another, and shared some basic intuitions. 'Rest' was also a point that FC loosely had in common with James Wilkes, the poet she met at a funding open day, and they liked each other too - so that started to become the topic of the project. Moreover, FC was only in that room because she had some support from Durham University to put something together for The Wellcome Trust Hub award which is to say, the support, like the desire for collaboration, came before the question. DF assembled the urban brain project (see Chapter 3) with Nikolas Rose and Ilina Singh because the three of them were thinking through their shared intuitions on sociology and biology; again, the decision to collaborate on these intuitions came before the identification of the actual research question. The point is that there is no right way to develop an interdisciplinary collaboration, and no correct order that the different elements must come in - interdisciplinary projects, like all intellectual work, are always loose assemblages with strange temporalities. If you have a really good idea, and you need someone from another discipline to help you with it - that's great. But don't be anxious if you just want to collaborate because you like someone, or you might get some support from your university if you do. The problem will emerge: there's no particular reason it has to emerge first.

Except where otherwise noted, this work is licensed under a Creative Commons Attribution 3.0 Unported License. To view a copy of this license, visit https://creativecommons.org/licenses/by/3.0/ 